\title{
Double Hough transform for estimating the position of the mandibular canal in dental radiographs
}

\author{
Darian Onchis-Moaca, Simone Zappalá, Smaranda Laura Goţia and Pedro Real
}

\begin{abstract}
In this work, a multiple generalised anisotropic Hough transform (AGHT) is used to detect the mandibular canal in dental panoramic radiographs.

The proposed method relies on a sequential application of the Hough transform that we call double Hough transform. The recognition of the mandibular canal is based on a double template matching compared with the clinical detection using the fact that the shape of the mandibular canal is usually the same and it is situated inside the mandibular bone.

The experiments performed on real orthopantomographic images shown that the risk of false detection is significantly decreased, while the recognition is not affected by occlusion and by the presence of additional structures e.g. teeth, projection errors.
\end{abstract}

\section{Introduction}

From a clinical point of view, the marking of the mandibular canal is useful in detecting the nerve for inferior teeth called inferior dental nerve which is found inside it.

Darian Onchis-Moaca

University of Vienna, Faculty of Mathematics, Oskar-Morgenstern-Platz 1, Vienna, Austria, email: darian.onchis@univie.ac.at

Simone Zappalá

University of Vienna, Faculty of Mathematics, Oskar-Morgenstern-Platz 1, Vienna, Austria, email: simone.zappala@univie.ac.at

Smaranda Laura Goția

Victor Babes University of Medicine and Pharmacy, Department of Physiology, Eftimie Murgu Street 2, Timisoara, Romania, e-mail: lauragotia@yahoo.com

Pedro Real, University of Seville, Department of Applied Mathematics I, Av. Reina Mercedes, Seville, Spain, e-mail: real@us.es 
While there are many research studies trying to visually identify the mandibular canal eg. [Atieh(2010), Mehra and Pai(2012), Jhamb et al.(2009)Jhamb, Dolas, Pandilwar and Mohanty] or to mark the canal by searching the whole image, like in [Onchis et al.(2015)], in this paper, we propose a double application of the generalized anisotropic Hough transform, first used to detect a part of the mandibular bone and to restrict the search area, followed by second application in the detection for marking the mandibular canal. This procedure decreases the risk of false detection of the mandibular canal by focalizing on the exact area where the canal is situated. The method is based on template matching of a shape which can be found in other zones of the dental radiography, representing other anatomical features.

The the Hough transform is a popular technique to extract features from an image. The method was patented in 1962 [Hough Paul(1962)] for the detection of lines in photographs. The functioning of the algorithm lies in a proper choice of the parameters space for the set of lines on the plane.

In order to develop a method for the recognition of a generic template in an image, [Ballard(1981)] used the following parameters for a shape:

$$
a=\{y, S, \theta\}
$$

where $y=\left(x_{r}, y_{r}\right)$ is a reference point to represent the translations, $S=\left(S_{x}, S_{y}\right)$ are scale values for the orthogonal shearing deformations, and $\theta$ is an angle that represents the rotations.

The reference point $y$ is described in terms of a table, called the R-table of the template, of possible edge pixel orientations. The other parameters are described in terms of transformations of the aforementioned table.

The key for generalizing the Hough transform is to use the directional information. Given a template, i.e. a set of boundary points $\left\{x_{B}\right\}$, a reference point $y$ is chosen. After the discretization of the straight angle through a uniform partition $\{0, \Delta t, 2 \Delta t, \ldots, N \Delta t\}$, for every boundary point the tangent direction $\phi\left(x_{B}\right)$ is computed, then $r=y-x_{B}$ is stored in the $n$th bin of the R-Table if $\bmod \left(\phi\left(x_{B}\right), \pi\right) \in$ $[(n-1) \Delta t, n \Delta t)$ as in [Onchis et al.(2015)]. Given this simple structure, with the use of rotation and shearing operators, i.e. $\operatorname{rot}_{\theta}$ and $d e f_{S}$, we can build the following procedure to detect the template in the set of edge points of any image. 
Data: IMG: Image

$\mathrm{R}-\mathrm{T}$ : R-Table of a template

$\{(S, \theta)\}$ : Set of scale and rotation parameters

WHERE:subregion of IMG

Result: Pattern Localization

Compute $\left(x_{e}, \phi\left(x_{e}\right)\right)$ edge points and their gradient;

for each $(S, \theta)$ do

for each $x_{e}$ do

Find $n \Delta t$ s.t. $\operatorname{def}_{S}\left(\operatorname{rot}_{\theta}\left(\phi\left(x_{e}\right)\right)\right) \in n \Delta t$;

for each $r \in n \Delta$ do

Compute $y_{(S, \theta)}^{\prime}=x_{e}+\operatorname{def}_{S}\left(\operatorname{rot}_{\theta}(r)\right)$;

if $y^{\prime} \in W H E R E$ then

Report an occurrence of $y_{(S, \theta)}^{\prime}$.

end

end

end

Select $y_{(S, \theta)}$ with higher occurrence ;

end

return $y$ with higher occurrence;

Algorithm 1: Hough_Recognition

In this way, we find the edge point that satisfy the non-analytic version of

$$
f(x, a)=0
$$

and

$$
\frac{\partial f}{\partial x}(x, a)=0
$$

The space of occurrences for the reference point is called Accumulator Space. Regarding complexity concerns of the algorithm, we point out that all of the 3 nested for loops could be parallelised.

\section{The recognition procedure}

Problems with AGTH recognition may arise when we have to deal with real images which could be corrupted by noise. That is the case of radiography where the structure of a bone is not well defined and where some part of the bone which can be disguised with unwanted details.

The position of the mandibular canal is described by medical indications as follows: the canal starts at the mandibular foramen in the middle part of the vertical ramus. It continues through the mandibular bone and ends in the menton foramen between apexes of the two inferior premolars. 
With this indicaton we can roughly compare the position of the canal against the barycenter of the mandible. This is how the doctor's mind work, by focusing on the interest area representing the horizontal ramus of the mandibular bone (first Hough transform) and recognizing a pattern which represents the mandibular canal (second Hough recognition). This is the algorithm that we want to mimic.

As shown below, the shape of the canal can become misleading if we analyze radiograph of a patient who has lost some teeth.

Any surgical intervention in the mandibular area must prevent any nerve injury. The injury of the nerve would result in prolonged local and lower lip anesthesia for a minimum period of six weeks. Estimating the position of the mandibular canal means knowing the position of the nerve and by this the surgeon can estimate the risks and to adapt the surgical procedure to the individual case.

In our test, we used Figure 1 to extract the template of the canal. The recognition

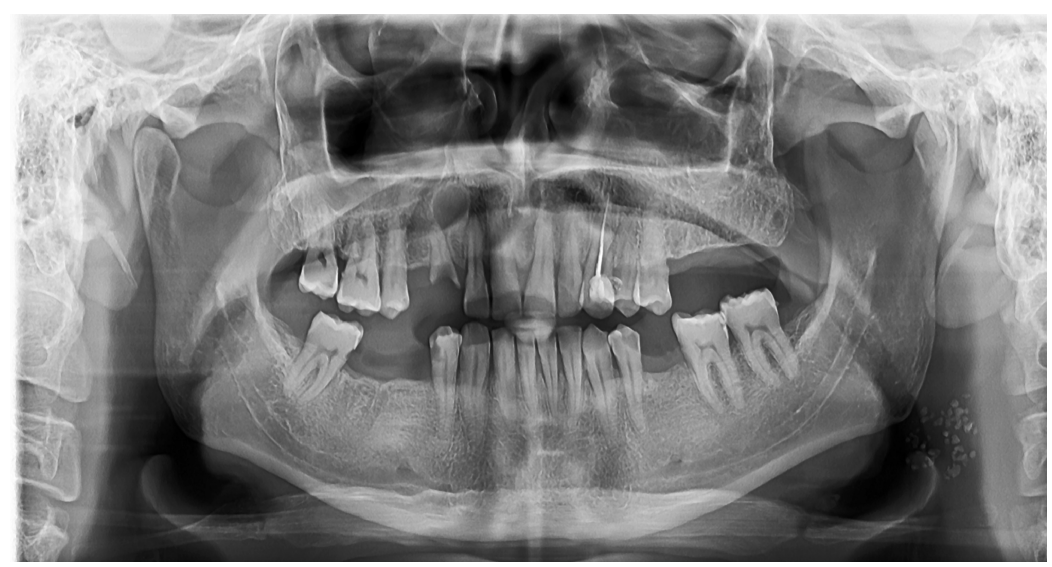

Fig. 1: Typical panoramic radiography

performs well on the same panoramic radiography as it can be seen in Figure 4, but the aforementioned image is in a critical situation: the patient has only one molar on the right part of the mandible.

When the mandible is edentated, without any further restriction, the canal could not be recognized anymore because the AGHT algorithm matches the template in Figure 2 with the top part of the alveolar process, the part of the bone where the teeth should be. It happens because the process is detected with a thicker edge than the canal, but has the same gradient direction and shape, so in the recognition process it will have more importance. This undesired, unavoidable, matching has been soften by using the modulus- $\pi$ direction of the gradient: the canal, as modeled in Figure 2 , looks like the empty space in an edentated mouth; by using the modulus we remove every information about the inside-outside of the model. This seems the best choice for the case of the mandibular canal, a poorly defined region of the mandible enclosed by a slightly brighter contour. 
This problem is related to how the AGHT is implemented: one of the weakness of this transform is that the accumulation space does not carry any information about the position in space of the template nor the mutual relation of different shape in the image. We identify two ways to overcome this problem:

- Manual solution: the user should restrict the area to be investigated manually through anatomical information given beforehand.

- Double-automatic solution: after a first, coarse and less accurate search for the mandible template through AGHT, the area to be investigated for the mandibular canal is automatically restricted.

We used the second method as described in the sequel.

\section{The double Hough transform method}

The canal template described in Section 2 has the following characteristics: it is a connected, compact and simply connected region of the plane. Therefore, after the binarization of the template, we can run a contour-following algorithm to detect the boundary points. This way, one could reach the first purpose for the proposed pattern recognition: to obtain an easy manipulable set of data samples.

The sorted array of boundary point that we obtain through a contour-following algorithm is well suited for the double Hough transform. As mentioned before, we do not need an accurate detection of the mandible, because this pattern belongs to the high level set of structure in the hierarchy of the image. In this way we can easily

- Compute the gradient of a boundary point knowing its neighbourhood

- Subsample the high level templates in a set of equally spaced point
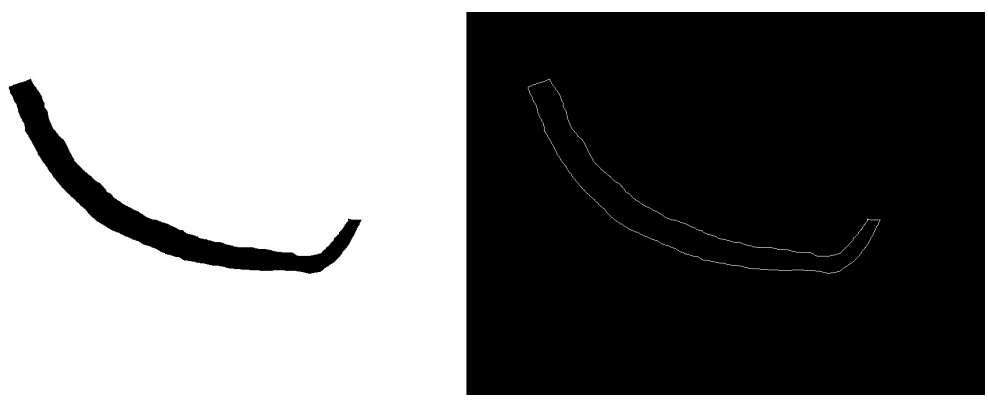

Fig. 2: Canal and its contour

The same technique cannot be applied directly to a panoramic radiograph. Edge points of are the result of an high pass filter applied to the image. 
We find out that common edge detector filters such as Canny, Sobel, etc., fail. Radiographs are spurious images which contain a great amount of unwanted details. So, we concentrated our work on the choice of the good parameters for the detection of the edges of the teeth (for a good survey see [Gráfová et al.(2013)Gráfová, Kašparová, Kakawand, Procházka and Dostálová]). This means the proper choice of the variance in the Gaussian filter and the threshold parameter.

The processes of low pass filtering and thresholding, cancel the mandibular canal from the image; so we have to create an ad hoc method for detecting the edges. It can be seen from Figure 1 that mandibular canals are drawn by two bright gray curved lines in a darker gray background; the good point is that the orientation of the canal is steady. Therefore, the natural idea is to use a high pass anisotropic filter mask adjusted on the shape of the mandibular canal.

To calculate the gradient needed in the implementation of the AGHT we used a Sobel mask for both the boundary and the edge points to have consistency in the calculation.

After the calculation of the barycenter of the template, for the construction of the R-table we chose to store $r=y-x_{B}$ in Cartesian coordinates to follow the natural discretization introduced by an image. This also helps us to understand the worst case scenario: after the recognition process the accumulator space will be a matrix with the same dimension of the image so we could print it on screen in grayscale to understand how the error spread and which other shape can be disguised as a mandibular canal.

We increment the accumulator space as in [Onchis et al.(2015)].

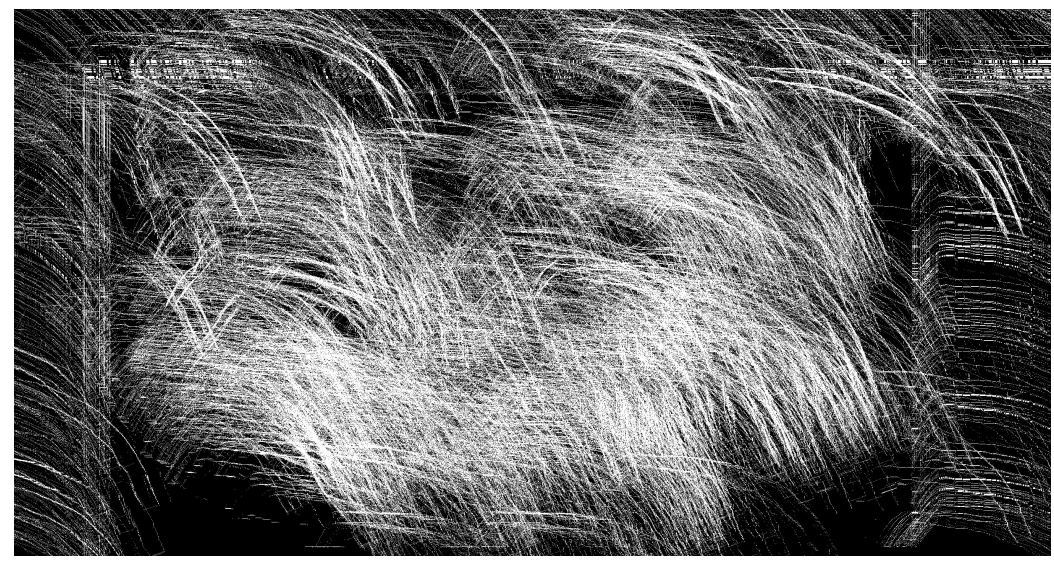

Fig. 3: Accumulator Space for Canal recognition

The last remark should be about the parameters expressed in (1). We have not used the rotational parameter $\theta$ inasmuch as every panoramic radiography is taken with the patient's head fixed. The important parameter is $S=\left(S_{x}, S_{y}\right)$ which helps us to reconstruct the anatomical difference among human beings. In this way, it is 
possible to find the best deformed version of the template in Figure 2 that matches the canal in the panoramic radiography under analysis.

We sum up in Algorithm 2 all the remarks we expressed in the previous section.

Data: IMG: Image

TMP_C: Mandibular Canal

TMP_M: Mandible

$\left\{\left(S_{x}^{C}, S_{y}^{C}\right)\right\}$ Possible Canal Scales

$\left\{\left(S_{x}^{M}, S_{y}^{M}\right)\right\}$ Possible Mandible Scales

Result: Template Localization

$\left\{x_{B}^{M}\right\}=$ Contour-Following(TMP $\_$M);

$\mathrm{R}-\mathrm{T} \_\mathrm{M}=\mathrm{R}-\mathrm{Tab} l \mathrm{e} \_\mathrm{Build}\left(\operatorname{subsample}\left(\left\{x_{B}^{M}\right\}\right)\right)$;

$y^{M}=$ Hough_Recognition(IMG,R-T_M, $\left\{\left(S_{x}^{M}, S_{y}^{M}\right)\right\}$, IMG);

Select A $\_$M an Area around $y^{M}$;

$\left\{x_{B}^{C}\right\}=$ Contour - Following $($ TMP_C $)$;

R-Table_C = R-Table_Build $\left(\left\{x_{B}^{C}\right\}\right)$;

$y^{C}=$ Hough_Recognition(IMG,R-T_C, $\left\{\left(S_{x}^{C}, S_{y}^{C}\right)\right\}$,A_M);

return $y^{C}$

\section{Results}

In this section, we present the experimental results of the proposed algorithm.

The first test is to search for the template extracted from Figure 1 in the same image. The overlapping is perfect even without the double technique. The same argument can be brought forward for the flipped template, since there is no significant anatomical difference between the left and the right side of the same patient.

The approach proposed in this paper is based on a Double-Hough transform, named in this way because it searches for the whole mandible to find the area of interest, then the AGHT recognition process for the canal is performed.

This process is possible because the canal lies in the center of the mandible, so their reference points (their barycenters) are really close. After the detection of reference points for the mandible $y_{M}=\left(x_{M}, y_{M}\right)$, we force the reference points of the canal to be in the square window $\left[x_{M}-50, x_{M}+50\right] \times\left[x_{M}-50, x_{M}+50\right]$, with underlining unit of measure the pixel (Figure 6 and 8 ).

It is easily observed in the Figure 9 that if the patient has all his teeth, the arearestriction process is not mandatory for the canal recognition. 


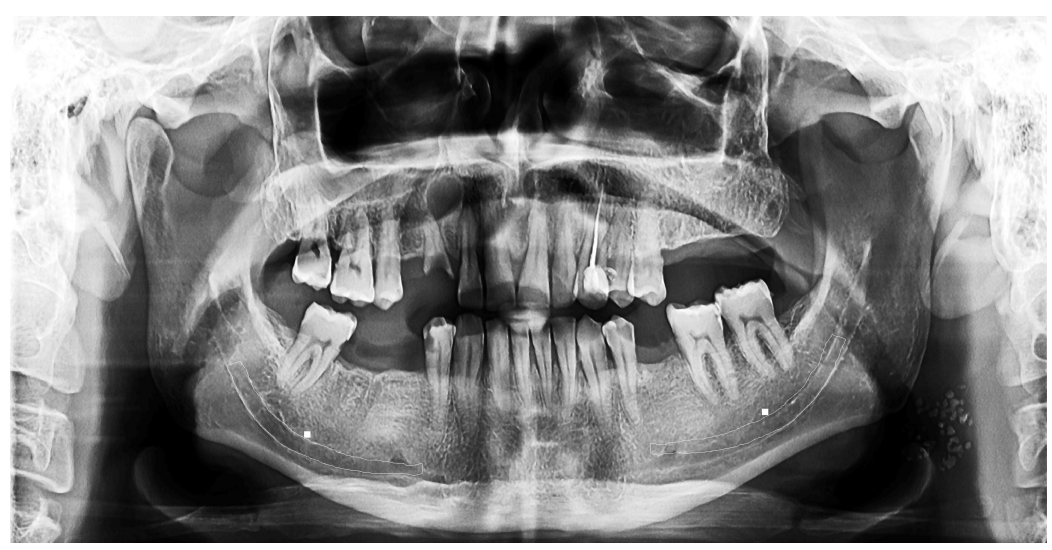

Fig. 4: Perfect matching of the template on the original image. Optimal matching of the flipped-left template

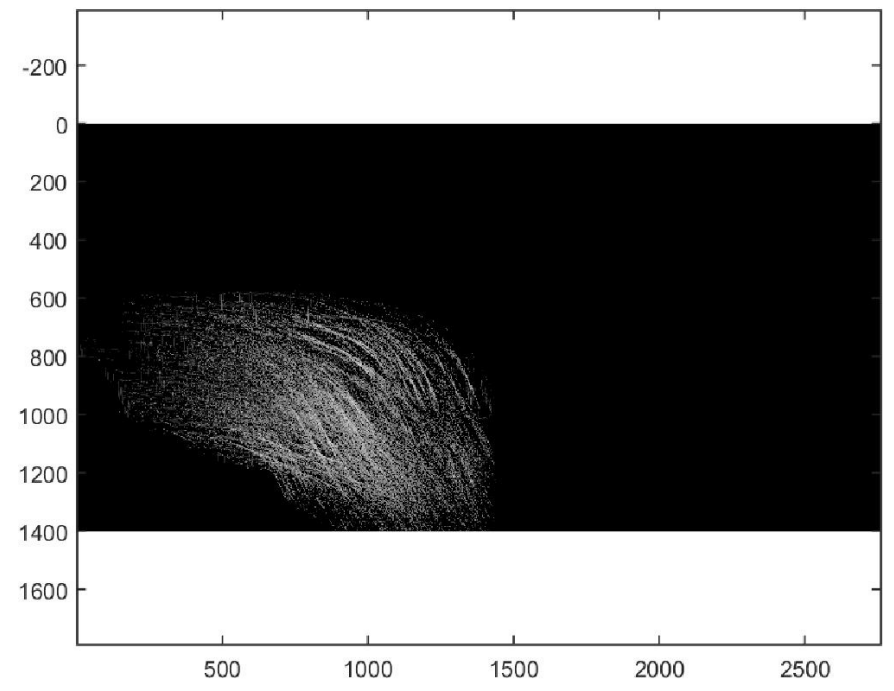

Fig. 5: Accumulator space for mandibular canal. Restriction to bottom-left part of picture 6 


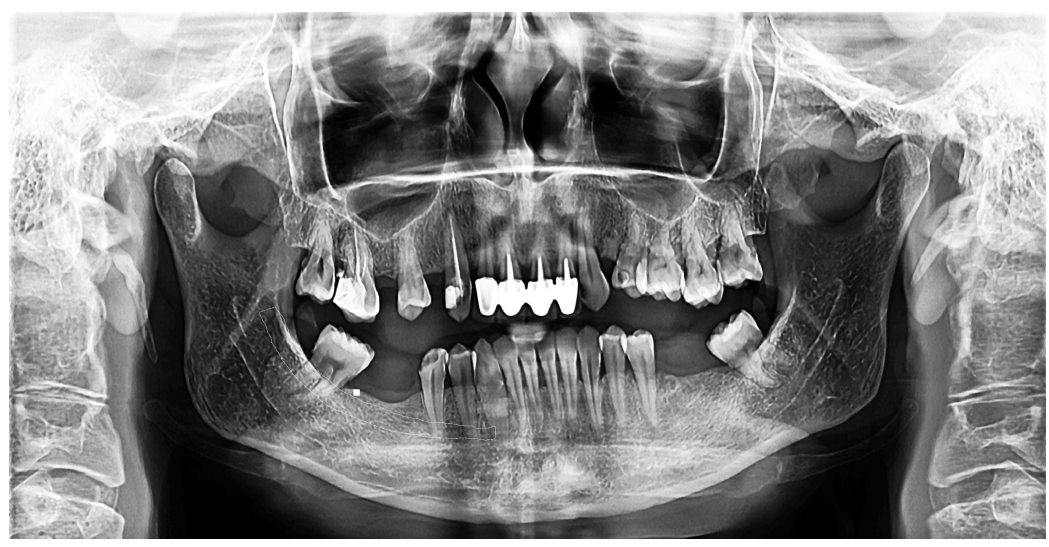

Fig. 6: Hough recognition without area restriction.

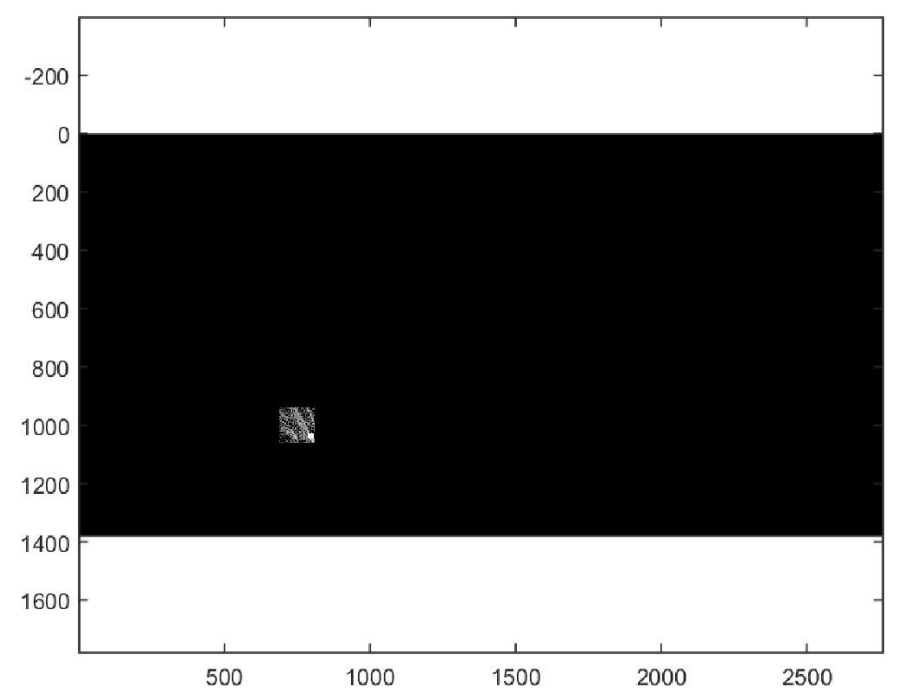

Fig. 7: Accumulator space for mandibular canal. Double-Hough restriction 


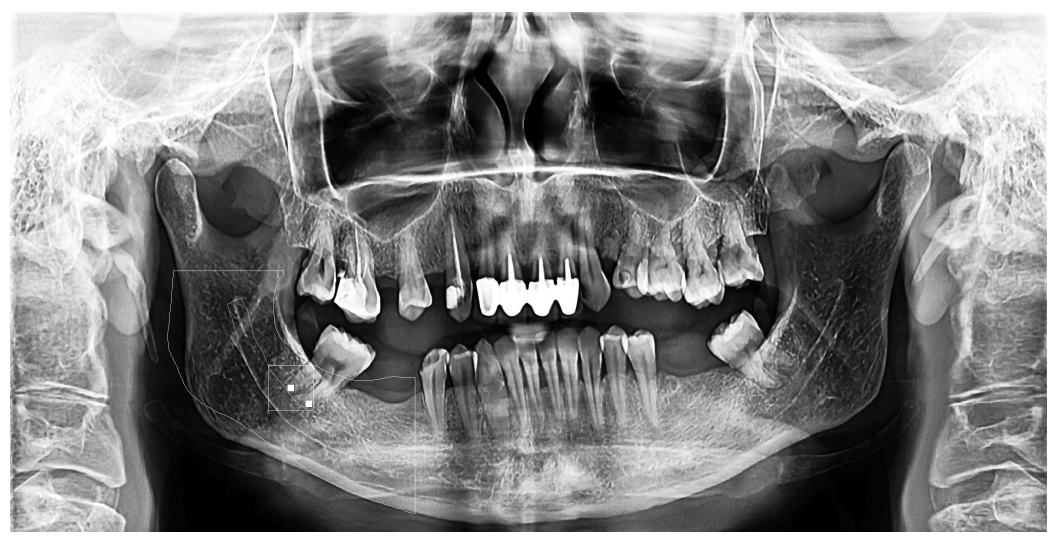

Fig. 8: Double-Hough solution through area restriction.

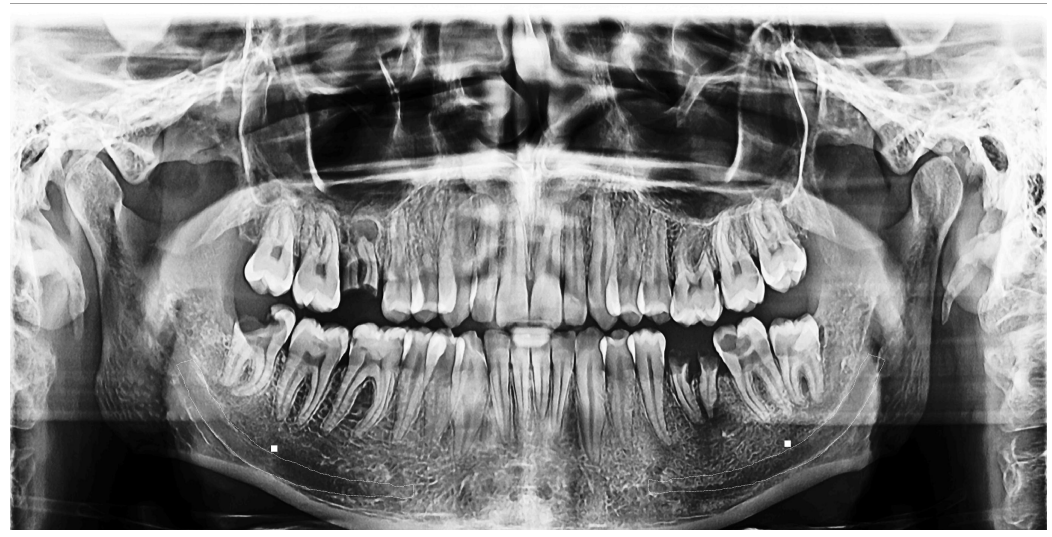

Fig. 9: Recognition of the template in figure 2 with scale parameters $\left(S_{x}, S_{y}\right)=(1.1,1.15)$

\section{Conclusions}

In this work, we used the double AGHT for the detection of the mandibular canal in a panoramic radiograph. We had to deal with different shapes which can be mistaken with each other, such as the top of the mandible and the mandibular canal.

We wanted to restrict the area to be analyzed using medical information with an automatic strategy.

Therefore, we used an automatic selection based on the mutual relation of different patterns in an image. 


\section{Acknowledgments}

The first two co-authors gratefully acknowledge the support of the Austrian Science Fund (FWF): project number P27516.

\section{References}

[Atieh(2010)] Atieh, M.A., 2010. Diagnostic accuracy of panoramic radiography in determining relationship between inferior alveolar nerve and mandibular third molar. Journal of oral and maxillofacial surgery $68,74-82$.

[Ballard(1981)] Ballard, D.H., 1981. Generalizing the Hough transform to detect arbitrary shapes. Pattern recognition 13, 111-122.

[Feichtinger and Strohmer(1998)] Feichtinger, H.G., Strohmer, T., 1998. Gabor analysis and algorithms: Theory and applications. Birkhäuser.

[Gráfová et al.(2013)Gráfová, Kašparová, Kakawand, Procházka and Dostálová] Gráfová, L., Kašparová, M., Kakawand, S., Procházka, A., Dostálová, T., 2013. Study of edge detection task in dental panoramic radiographs. Dentomaxillofacial Radiology 42, 20120391.

[Hough Paul(1962)] Hough Paul, V.C., 1962. Method and means for recognizing complex patterns. US Patent 3069654.

[Illingworth and Kittler(1988)] Illingworth, J., Kittler, J., 1988. A survey of the Hough transform. Computer vision, graphics, and image processing 44, 87-116.

[Jhamb et al.(2009)Jhamb, Dolas, Pandilwar and Mohanty] Jhamb, A., Dolas, R.S., Pandilwar, P.K., Mohanty, S., 2009. Comparative efficacy of spiral computed tomography and orthopantomography in preoperative detection of relation of inferior alveolar neurovascular bundle to the impacted mandibular third molar. Journal of Oral and Maxillofacial Surgery 67, 58-66.

[Karlheinz(2001)] Karlheinz, G., 2001. Foundations of time-frequency analysis. Birkhäuser.

[Mehra and Pai(2012)] Mehra, A., Pai, K.M., 2012. Evaluation of Dimensional Accuracy of Panoramic Cross-Sectional Tomography, Its Ability to Identify the Inferior Alveolar Canal, and Its Impact on Estimation of Appropriate Implant Dimensions in the Mandibular Posterior Region. Clinical implant dentistry and related research 14, 100-111.

[Onchis et al.(2010)Onchis, Real and Gillich] Onchis, D.M., Real, P., Gillich, G.R., 2010. Gabor frames and topology-based strategies for astronomical images, in: Proceedings of CTIC 2010 Computational Topology in Image Context, Chipiona, Spain, pp. 159-166.

[Onchis et al.(2015)] Onchis, D.M., Zappala, S., Gotia, S.L., Real, P., Pricop, M., 2015. Detection of the mandibular canal in orthopantomography using a Gabor-filtered anisotropic generalized Hough transform. Pattern Recognition Letters In press. 